\title{
Local Dislocation Creep Accommodation of a Zirconium Diboride
}

\section{Silicon Carbide Composite}

\author{
M.W. Bird ${ }^{\mathrm{a}}$, T. Rampton ${ }^{\mathrm{b}}$, D. Fullwood ${ }^{\mathrm{b}}$, P.F. Becher ${ }^{\mathrm{c}}$, K.W. \\ White \\ ${ }^{\mathrm{a}}$ Department of Mechanical Engineering, University of Houston, Houston, TX, USA \\ ${ }^{\mathrm{b}}$ Department of Mechanical Engineering, Brigham Young University, Provo, UT, USA \\ ${ }^{c}$ Department of Materials Science and Engineering, University of Tennessee, Knoxville, TN, USA
}




\section{Abstract}

A grain boundary sliding creep mechanism, accommodated by "mantle" dislocation activities, is shown to allow for large strain $(\varepsilon>0.08)$ during the creep of a $\mathrm{ZrB}_{2}-20 \% \mathrm{SiC}$ composite at $1800^{\circ} \mathrm{C}$. We characterized the local grain deformation behavior using high resolution electron backscatter diffraction (HREBSD) microscopy and an indentation deformation mapping (IDM) technique. Deformation gradients near grain boundaries (“mantle") produced average geometrically necessary dislocation (GND) densities of $3 \times 10^{11}$ $4 \times 10^{12} \mathrm{~cm}^{-2}$, about one order of magnitude above that of the grain interiors ("core"). A deviation from single crystal grain core deformation defines the mantle where excess GND's accommodate the grain deformation gradient. Evidence supporting grain boundary sliding as the primary contribution to the creep strain appears in our earlier publication, but we show here the role of dislocations in the deformation of the grain mantle as the rate-controlling accommodation step. 


\section{Introduction}

Extreme temperature environments $\left(\mathrm{T}>1500^{\circ} \mathrm{C}\right)$ require metal or metal compounds with melting points greater than $3000^{\circ} \mathrm{C}$. [1] [2] A survey of materials meeting this one requirement is limits the list to precious refractory metals, oxide, boride, carbide and nitride ceramic systems. Group IV borides, select group IV and V carbides and nitrides meet the high melting point criteria for such extreme environments. [1] [2] [3] Zirconium diboride compounds offer a unique combination of high melting points, high thermal conductivity, low density and thermodynamic stability which make such materials candidates for aerodynamic applications ranging in temperature between $1500-2200^{\circ} \mathrm{C}$ in oxidizing environments. Mechanical behavior transitions are to occur from predominately linear elastic to viscoplastic behavior. $\mathrm{ZrB}_{2}-20 \% \mathrm{SiC}$ bending creep experiments conducted between 1400 and $1800^{\circ} \mathrm{C}$ identified two distinct material behaviors: for $\mathrm{T}<1500^{\circ} \mathrm{C}$ diffusion creep and grain boundary sliding, while beyond $1600^{\circ} \mathrm{C}$ grain boundary sliding at increased steady state strain rates of $10^{-9}-10^{-5} \mathrm{~s}^{-1}$ were observed. [4] Continued creep research on these composites have shown large accumulated macroscopic creep strains $(>10 \%)$ with $<10 \%$ cavitation leaving the post-creep microstructure very similar to its initial state. This, along with relatively low stress exponents, has focused our attention toward grain boundary sliding models, linked with a necessary dislocation accommodation process.

Langdon [5] proposed grain boundary sliding as an independently operating creep mechanism while maintaining a constant shape. [5] [6] Later, Ashby and Verrall proposed a 2-D neighbor-switching model with diffusion creep accommodating the small grain shape changes necessary for the sliding event. Gifkens [7] [8] expanded on this notion, considering dislocation accommodation within a "mantle" region of the grain while leaving the "core" relatively free of 
dislocation activity. Combinations of grain boundary sliding and any affiliated rotations were needed for maintaining microstructural continuity while permitting large relative grain translations. A slip-dominated deformation process with increasing stress, decorates the mantle zone with both statistically stored (SSD) as well as geometrically necessary dislocations (GND's) to satisfy the deformation compatibility requirements with the grain core. [7] This model promotes increased GND (mantle) importance with decreasing grain size. Both models require an increased mobile dislocation density in the mantle boundary over that of the grain core. This accommodation event will be shown to involve local grain deformations of the order of grain geometric parameter, $y$, as shown in Figure 1.

Polycrystalline materials are plastically inhomogeneous, causing incompatible deformation between grains oriented with different critical resolved shear stresses or the presence of a second phase. [9] GND's are required for microstructure compatibility in addition to the accumulating SSD's. Both SSD and GND dislocations can be individually inspected using transmission electron microscopy. [10] However, such studies typically cover a tiny specimen area, and sample preparation can remove or modify many of the defects being studied. At a higher length scale, X-ray techniques are often used for quantifying the GND densities of a plastically deformed polycrystal. These methods rely upon a continuum field of dislocations. At a given length scale, the net effect of GNDs within a virtual Burgers circuit distorts the crystal lattice. The rotational component of this distortion is then measured, and the GND content inferred; SSD's provide no net contribution to the distortion. However, the spatial resolution of the X-ray techniques is of the order of several microns, and is therefore not suitable for the more detailed study required for this paper.

On the other hand, Electron Backscatter Diffraction (EBSD) and post processing Orientation Imaging Microscopy (OIM) routines provide a higher resolution view of the GND 
fields. [11] [12] [13] [14] These methods characterize the Kikuchi pattern distortion relative to a perfect, strain-free, pattern of the same orientation, or relative to a pattern from elsewhere on the specimen. [12] [13] For polycrystalline materials the GND density tensor is calculated based on Nye's relationship following [15] [16]

$$
\alpha_{i k}=e_{j l k}\left(\frac{\partial \beta_{i j}}{\partial x_{l}}\right)=\nabla \times \beta
$$

where the dislocation tensor $\alpha_{i k}$, defined to be the curl of the elastic distortion tensor $\beta_{i j}=\frac{\partial u_{i}}{\partial x_{j}}$, includes both rigid rotation and strain components. [12] [14] $e_{j l k}$ is the Levi-Civita symbol (or permutation tensor), and the usual Einstein summation convention is observed for repeated indices within a given term. The GND density on a given slip system, $\rho^{(m)}$, contributes to the total Nye tensor via the equation: $\alpha_{i j}=\sum_{m} \rho^{(m)} b_{i}^{(m)} v_{i}^{(m)}$, where $b$ is the relevant Burgers vector, and $v$ is the corresponding unit vector in the line direction. Since OIM is a surface characterization technique, the derivatives required for Equation 1 are only available in the 1 and 2 directions. Hence the only fully determinable components of the Nye tensor are $\alpha_{13}$, $\alpha_{23}$ and $\alpha_{33}$. This corresponds to the net Burger's vector of a circuit normal to the sample surface. [14] However, several other components of the Nye tensor can be estimated if strain gradients are neglected in the presence of considerably larger rotation gradients. [14] If all available distortion terms are included in the GND estimate, and the L1 norm (the sum of component absolute values) of the resulting Nye tensor is scaled to compensate for unknown components, a more accurate picture of the GND content can be obtained [17]. Therefore, total GND concentration can be approximated with reasonable accuracy as long as deformation is considered uniform through the sample thickness. 
The present work experimentally separates the deformations associated with translation from that associated with rotation, and models mantle deformations as a critical step in accommodating grain boundary sliding creep, in the context of Orowan's [18] classical representation of plastic strain

$$
\gamma=\rho^{(m)} b \bar{x}
$$

The plastic shear strain, $\gamma$, describes the total number density of mobile dislocations, $\rho^{(m)}$, moving on a slip plane an average distance $\bar{x}$ and $b$ the Burgers vector. We consider two independent methods for probing the microstructure, particularly, understanding the role of the mantle defects in this accommodating step, as distinguished from the grain core behavior. First, deformation mapping using EBSD and improved OIM techniques were employed for spatially quantifying GND densities. To the authors' knowledge, this advanced microscopy technique has not been attempted for quantifying creep deformation structures in ceramics, including these diboride systems. As a second approach, we introduce our version of earlier scribe experiments [19] , referred to here as the Indentation Deformation Mapping (IDM) method, which allows for the assessment of grain-to-grain deformation and rotation. The deformations obtained by this IDM method will be interpreted within the context of Orowan's model and the EBSD evidence of dislocation density distributions within the grains. .

\section{Experimental Methods}

\subsection{Materials and Processing}

ZrB2-20vol\% SiC billets were produced by Missouri University of Science and Technology (MST). MST billets were hot pressed in a similar manner to that described in [20]. $\mathrm{ZrB}_{2}$ powder (Grade B, H.C. Starck, Newton, MA) was $>99 \%$ pure with average particle size of $2 \mu \mathrm{m}$. SiC 
powder (Grade UF-10, H.C. Starck) was predominantly $\alpha$-SiC with purity of $98.5 \%$ and particle size of $0.7 \mu \mathrm{m} . \mathrm{B}_{4} \mathrm{C} / \mathrm{ZrB}_{2}$ powder (Grade HD-20, H.C. Starck) was also added to billets at $2 \mathrm{wt} \%$ to reduce $\mathrm{SiC} / \mathrm{ZrB}_{2}$ particle oxide surface films improving densification during pressing. Powders were ball milled with WC-6Co media, resulting in approximately 2.2 to 2.4 wt\% WC-6Co impurity concentrations based on true and theoretical density differences. Confirmation of impurity concentration by XPS analytical technique is reported elsewhere. [21] Additionally, Electron Dispersive Spectroscopy (EDS) confirmed the average $\mathrm{W} / \mathrm{Zr}$ atomic ratio. A maximum hot pressing temperature and pressure of $1950^{\circ} \mathrm{C}$ and $32 \mathrm{MPa}$, respectively, with heating rates of $30^{\circ} \mathrm{C} / \mathrm{min}$ up to $1650^{\circ} \mathrm{C}$ and $90^{\circ} \mathrm{C} / \mathrm{min}$ up to $1950^{\circ} \mathrm{C}$ were employed.

\subsection{Characterization}

Quantitative microscopy was completed using a scanning electron microscopy (LEO 1525 FE SEM SE Detector and FEI Quanta 400 SEM BSE and SE Detector). Fine scan Electron Backscatter Diffraction (EBSD) was conducted on a single crept and uncrept specimen, for a total of two samples, using a Hikari camera and Octane Energy Dispersive Spectroscopy (EDS) detector mounted on a Philips XL-30 FEG SEM. A survey window of approximately $15 \mu \mathrm{m} x$ $15 \mu \mathrm{m}$ with a spot size of $5 \mathrm{~nm}$, at a step size of $0.050 \mu \mathrm{m}$, surveying approximately $10-30 \mathrm{ZrB}_{2}$ grains, and $1 \times 1$ binning was used for collecting EBSD generated Kikuchi patterns of both $\mathrm{ZrB}_{2}$ and SiC phases. [22] Kikuchi patterns were saved as Bitmaps at each point for post processing. Samples were prepared using SiC grinding paper and polished to $0.25 \mu \mathrm{m}$ diamond suspension, then finished with $0.05 \mu \mathrm{m}$ colloidal polish on a vibratory mat. Polishing slightly recessed the softer $\mathrm{ZrB}_{2}$ grains as compared to the harder $\mathrm{SiC}$ phase (Figure 2).

Orientation Imaging Microscopy (OIM) data collection and analysis of $\mathrm{ZrB}_{2}$ and $2-\mathrm{H} \alpha-\mathrm{SiC}$ Kikuchi patterns was completed using the EDAX TSL OIM Data Collection/Analysis V5.3 software. 
A typical SEM and OIM image reconstruction shown in Figure 2 provides the necessary Euler angle descriptions for crystallographic real space interpretation, including grain size, shape and crystal direction statistics. Furthermore, this figure notes the sample reference frame with respect to the loading axis and crystallographic real space are shown. Kernel Average Misorientaiton (KAM) analysis was used for qualitatively assessing the spatial distribution of regions containing local lattice distortion. [23] Second nearest neighbor, inclusive, kernel averaging was implemented for $0-2^{\circ}$ of local misorientation with an angular resolution of $0.5^{\circ}$.

Using relation (1) and the associated discussion, and only considering the rotation components of the lattice distortion, the kernal average misorientation, $\theta$, can be directly linked to an estimate for GND density using the equation: $\rho_{G N D}=2 \theta / L b$, where $\mathrm{L}$ is the step size between second nearest neighbor points, and $b$ is the size of the Burgers vector [24]. This approximation assumes that all distortion is caused by screw dislocations, and therefore, generally underestimates this distortion. Alternatively, high-resolution EBSD, seeks to determine the full elastic distortion tensor, $\beta$, required to transform a reference structure to the local structure under consideration, by comparing the EBSD patterns of the two structures using convolution techniques. [13] [25] [26] [27] The Nye tensor requires derivatives of the distortion tensor, in turn, obtained from the strain gradient measured by adjacent EBSD patterns. [28] As mentioned, this approach only provides derivatives in the plane of the specimen surface, resulting in an incomplete Nye tensor. Nevertheless, this incomplete tensor is used to estimate the total GND density by adding the absolute values of the components, and scaling to compensate for missing components. [17] Using methods and code developed at BYU, this approach was used for the quantitative estimates of GND density presented in the rest of the paper. 
Our novel Indentation Deformation Mapping (IDM) method places an array of nanoindentations as microstructure-scale markers to track local creep deformations on the tensile face of a flexure creep specimen. Cube-corner nano -indentations were made using the MTS Nano Indenter XP (MTS, Eden Prairie, MN) in a $1 \times 100$ indent array, positioned parallel with the bending stress axis and within $250 \mu \mathrm{m}$ from the bending outer-fiber, and at an indentation depth of $200 \mathrm{~nm}$ and $1.5 \mu \mathrm{m}$ spacing. No cracking was observed at indentation depths less than 200 $\mathrm{nm}$. The indent array was imaged before and after the creep experiment using either secondary (Figure 3) or backscatter electron SEM detectors. The resulting images were analyzed using the binary threshold image analysis (Nikon Nis-Elements Documentation V3.22.00), to determine the following four local strain conditions. First, the total strain was calculated from indent horizontal displacement changes. Grain translation strains and grain rotation angles were measured between indent pairs of different grains. Finally, the grain deformation strains were measured from two indents within the same grain. Figure 3,

\subsection{Creep Experiment}

The vacuum-inert environment proved essential for preserving the polished specimen surfaces for subsequent analysis (graphite resistance vacuum furnace, (Centorr Vacuum Industries, Nashua, NH). Inert atmosphere was achieved by back filling 99.999\% Argon to an overpressure of $<3$ psig after evacuating to below 30 mTorr. Dead-load creep testing used $\alpha$-SiC 10/20 mm four-point bend fixtures with $6.35 \mathrm{~mm}$ diameter $\alpha$-SiC rollers. Prior to closing the furnace, before each run, a tungsten-filled tantalum cup placed on a graphite pedestal directly over the inner-span bending fixture provided the required load. Note, no real-time access to the specimen was possible for displacement measurements. However, final tensile and compressive strains were optically measured using methods detailed in [4] A total of two specimens (1.75 x 
$3.5 \times 25 \mathrm{~mm}$ ) were tested at $1800^{\circ} \mathrm{C}$ and applied stresses of $16 \mathrm{MPa}$ under these specialized conditions, and compared to our conventional creep data under conditions for verification and projection of the complete curves. [4]

\section{4. $\underline{\text { Results }}$}

\subsection{Vacuum Environment Creep}

Two flexure specimens, shown in Figure 4, were crept to outer fiber tensile strains of 15.6+/-1.5 and 8.1 +/- 1.0, and outer fiber compressive strains of $6.5+/-1.0$ and $3.8+/-0.4$, respectively. Because these strains were measured in the crept condition, an average creep rate (including primary and secondary creep strains) was used as a metric for comparing the instrumented and vacuum environment creep results. For the present case, average creep strain rates of $5.59 \times 10^{-6} \mathrm{~s}^{-1}$ and $4.50 \times 10^{-6} \mathrm{~s}^{-1}$ compare favorably with those instrumented strain rates of $5.41-5.99 \times 10^{-6} \mathrm{~s}^{-1}$ for equivalent temperature and stress conditions. These results suggest the vacuum creep process follows the creep behavior of the same composite under protective environment conditions [4] and primary and secondary (steady state) creep rates are nearly equivalent, consistent with the creep curves observed in [4].

\subsection{Indentation Deformation Mapping}

The IDM experiment provided insight into local $\mathrm{ZrB}_{2}$ grain deformation mechanisms, linking local micro-deformation to the large-scale creep behavior. Using these results, as well as the $\mathrm{SiC}$ particles as flags, local grain movement statistics were compiled for select grains from the IDM experiment and are summarized in Table 1. These measurements were used for assessing single grain mantle and core deformations according to Equation 1.

\subsection{High Resolution Electron Backscatter Diffraction}


Fine scan EBSD patterns were collected on a high strain ( $>8 \%$ tensile outer-fiber), Figure 4, specimen from both compression and tension regions. We observed creep asymmetry , where optically determined outer-fiber tensile strains were $\sim 2 \mathrm{x}$ those measured in compression. From reconstructed OIM images, we computed the total GND density corresponding to each pattern ( $x-y$ coordinate pair) from Equation 1, Figures 5 and 6 . We offer the following two observations: The SiC phase shows the highest GND density of $\sim 1 \times 10^{12} \mathrm{~cm}^{-2}$. Of critical importance to the present case however, the two grain boundary mantle types $\left(\mathrm{ZrB}_{2}\right.$ and $\mathrm{SiC}-\mathrm{ZrB}_{2}$ ) demonstrate densities one to two orders of magnitude above the core. That of the mantle is $1 \times 10^{11}-1 \times 10^{12} \mathrm{~cm}^{-2}$ while the core is found to be $1 \times 10^{9}-5 \times 10^{10} \mathrm{~cm}^{-2}$.

TEM research on $\mathrm{ZrB}_{2}$ powders and fully dense compacts show a wide range of dislocation densities. Goutier, et al. [29] observed significant plastic deformation, within $\mathrm{ZrB}_{2}$ grains, accommodating the thermal strains developed from spark plasma sintering of $\mathrm{ZrB}_{2}-\mathrm{ZrC}$ composites. In the presence of large thermal gradients, a dislocation density of approximately $10^{-9}-10^{-10} \mathrm{~cm}^{-2}$ was found for deformed $\mathrm{ZrB}_{2}$ grain cores neighboring $\mathrm{ZrC}$ grains. Mishra et al. [30] investigated the $\mathrm{ZrB}_{2}$ powder defect structures for three powder processing routes. Dislocation densities of $\sim 10^{8} \mathrm{~cm}^{-2}$ were measured for $20 \%$ of particles for traditional carbothermic and replacement reaction powder processing techniques. For the highly exothermic selfpropagating high temperature synthesis, $80 \%$ of particles had grain core dislocation densities of $10^{12} \mathrm{~cm}^{-2}$ suggesting accommodation plasticity within $\mathrm{ZrB}_{2}$ particles due to thermal gradients [30]. Other $\mathrm{ZrB}_{2}-\mathrm{SiC}$ and $\mathrm{ZrB}_{2}-\mathrm{MoSi}_{2}$ TEM studies have shown large isolated interphase dislocation densities contrasting with low dislocation density grain cores. [31] [32] Haggerty and Lee [33] observed easy glide and subsequent work hardening during compression experiments at $2050^{\circ} \mathrm{C}$ for single crystal $\mathrm{ZrB}_{2}$, suggesting low initial dislocation densities, followed by increasing statistically stored dislocations (SSD) density. We recognize these dislocations differ 
from the GND-type, however, the presence of grain boundaries in otherwise low-dislocation grain core regions would generate the necessary deformation strain gradients and, therefore, promote the increased GND density and subsequently the total dislocation density near the grain boundary. [9] We, therefore, feel that the present HREBSD method likely provides a reasonable approximation of the GND population for $\mathrm{ZrB}_{2}$.

\section{Analysis and Discussion}

Grain boundary sliding, as an individual deformation mode, has been widely linked with dislocation mechanisms as the accommodating step. Langdon [5] proposed grain boundary sliding as a primary deformation mechanism controlled by alternating dislocation glide and climb within the grain boundary dislocation network or along adjacent zones. Ball and Hutchinson [34] considered sliding of four-grain groups, accommodated by dislocation glide in favorably oriented grains. Gifkens [7] later proposed a sliding event similar to Ashby and Verrall's [35] model where deformation gradients respond to neighbor activity, leaving the mantle / core structure. [8] Therefore, we propose dislocation mechanisms as the local accommodation events, that may provide a necessary step to allow for the primary strain event, namely grain boundary sliding.

A revised GND rendering is shown in Figure 7, detailing only the $\mathrm{ZrB}_{2}$ GND magnitudes and spatial distributions; SiC GND calculations were ignored because of distorted EBSD patterns from polishing damage artificially inflating the total dislocation concentration. Bird, et al. [4] [36] has suggested that $\mathrm{SiC}$ deformation contributes $<5 \%$ to the macroscopic tensile creep strains, so we omit this from the forthcoming analysis.

We focus attention toward the one-order of magnitude increase in GND density

characteristic of the crept microstructures (Figure 7). Calculated GND densities of $\sim 10^{11} \mathrm{~cm}^{-2}$ are 
shown along both the $\mathrm{ZrB}_{2}-\mathrm{SiC}$ interphase and $\mathrm{ZrB}_{2}-\mathrm{ZrB}_{2}$ boundaries, in the hot-pressed microstructure, as a result of creep deformation during pressing. The deformation zones adjacent to all grain boundaries thicken with creep deformation from a range of 179 - $239 \mathrm{~nm}$ to 238 - $308 \mathrm{~nm}$. The observed GND density changes with distance into $\mathrm{ZrB}_{2}$ grains according to the Orowan [18] formalism from Equation 2, where $\bar{x}$ is taken as the distance from the grain boundary and $\rho_{m}$ the GND density in this gradient zone. We assumed GND's originate from the grain boundary and travel a distance away from the grain boundary (Figure 1) due to shear displacements required for accommodating the creep deformation incompatibilities in the polycrystalline composite.[7][8][9] Using linear regression, the Orowan model was fit to the calculated GND-distance relationship from ten random line profiles overlaid on the HREBSD rendering, Figure 8, assuming the total GND population is mobile. [9] These line profiles were placed originating from either the $\mathrm{ZrB}_{2}$ or $\mathrm{ZrB}_{2}-\mathrm{SiC}$ grain boundaries as shown in Figure 7. Although, the two slopes are not perfectly matched, this comparison with the Orowan model suggests constant strain across the grain, similar to single crystal deformation, with an observed deviation approaching $\sim 250 \mathrm{~nm}$. The constant strain region defines the grain core deformation region. The observed transition apparently represents the limit of a region adjacent to the grain boundary, following a different constant strain slope, we define as the mantle region. Our methods showed no statistically significant differences in the GND density profiles between the $\mathrm{ZrB}_{2}$ and $\mathrm{ZrB}_{2}-\mathrm{SiC}$ interphase boundaries, but have separated the datasets for completeness.

Local measurements of grain movements show that both grain deformations and rotations result from the sliding event. [7] [8] The creep model requires grain rotation as a response to stress relaxation ahead of a dislocation pileup at grain boundary triple points or flow along sliding interphase boundaries. Therefore, assuming hexagonal grain geometry and 
the presence of ledges along the sliding boundary, a minimum mantle thickness accommodating the deformation follows [37] :

$$
y=\frac{d}{2}(1-\cos \theta)
$$

where the mantle thickness, $y$, scales with the grain size, $d$, for a model hexagonal grain shape rotating through an angle $\theta$. Here, y represents the dislocation travel distance from the grain boundary inward toward the grain center, as a result of grain boundary sliding, Figure 1.[37] The maximum mantle thickness for our model hexagon approaches $y_{\max } \approx 0.067 \mathrm{~d}$ with a $30^{\circ}$ grain rotation. [8] The present IDM experiments find average $\mathrm{ZrB}_{2}$ grain rotations of $4.9^{\circ}$ with maximum rotations of $37^{\circ}$ and $0^{\circ}$, corresponding to average mantle thicknesses of $19 \mathrm{~nm}$ with a range falling between $154 \mathrm{~nm}$ and $0.40 \mathrm{~nm}$. Mantle thicknesses below one Burger's vector correspond to $\sim 1^{\circ}$ of grain rotation, for our $\mathrm{ZrB}_{2}$ grain sizes confining slip accommodation within the grain boundary plane. The distribution of mantle thicknesses suggests varying degrees of local mantle deformation in response to the macroscopic stress. In an effort to explain the mobile GND density plateau approaching the grain boundary region, as determined from HREBSD methods (Figure 8), we combine the mantle deformation model from ref [37] with Equation 2. Mantle mobile dislocation densities were calculated following a geometric model:

$$
\varepsilon=\frac{2 y}{d}=1-\cos \theta
$$

where all components of the normal strain, $\varepsilon$, appear in Equation 3. This normal strain represents the local grain shape changes needed for accommodating grain boundary sliding, which do not contribute to the overall specimen extension (or compression). If we include a Schmid orientation factor of $1 / 2$ we may relate the normal grain strain to a shear strain $\varepsilon=\frac{1}{2} \gamma$. Combining Equations 2 and 4 gives 


$$
\rho^{(m)}=\frac{2(1-\cos \theta)}{b \bar{x}}
$$

\section{(5)}

As with the HREBSD GND profiles, we take $\bar{x}$ as the average distance a dislocation travels from the grain boundary and becomes equal to y within the context of Equation 3, as outlined in ref [37] and Figure 1. It becomes apparent the defined accommodation strain (Equation 4) and average dislocation sweep distance (Equation 3) are dependent upon the geometric grain rotation and grain size. Therefore, Equation 5 suggests the mobile dislocation density should plateau under a normal grain size distribution within the defined mantle region. The computed dislocation densities (Equation 5) and the corresponding $\bar{x}(\mathrm{y})$ values (Equation 3 ), from the IDM measured grain rotations, are shown in Figure 9. We observed excellent agreement between our two methods for determination of average dislocation densities, where about $3 \times 10^{11} \mathrm{~cm}^{-2}$ compares well with $\sim 4 \times 10^{11} \mathrm{~cm}^{-2}$ for the IDM and HREBSD methods, respectively, below the apparent transition distance $(\bar{x})$ or mantle width $(y)$ equal to $250 \mathrm{~nm}$. Furthermore, we apply Equation 5 using grain size distribution from Figure 7, yielding excellent agreement with the GND densities computed using the HREBSD method (Figure 8). This confirms the onset of a separate deformation region (mantle region) having a one-order of magnitude larger defect density approaching the grain boundary. The HREBSD and IDM plateau GND density averages are statistically indistinguishable despite the larger creep strain for the HREBSD specimen. As a test, we compare our two methods using Ashby's GND analysis [9] for macroscopic deformation of polycrystalline materials, which predicts a larger HREBSD GND density than that of the IDM approach by a factor of 1.8. But, the close agreement we find between these two methods further emphasizes the role of this dislocation process as an accommodation mechanism rather than as a direct contribution to the macroscopic creep strain (Equation 5). We point out that 
our IDM and HREBSD results represent creep structures following 5 and nearly 8 hours of creep exposure, respectively, while the accommodation mechanisms have shown to generate a similar GND pattern.

Dislocation densities of the mantle estimated by both the IDM and HREBSD results show about an order of magnitude excess over the core (a factor of 13 and 12, respectively). The HREBSD GND densities deviate from the grain core Orowan model when approaching $\bar{x}<250 \mathrm{~nm}$ with an average $\rho_{G N D} \approx 4 \times 10^{11} \mathrm{~cm}^{-2}$; the theoretical IDM and HREBSD mantle widths are $295 \mathrm{~nm}$ and $304 \mathrm{~nm}$, respectively, having average grain sizes of $4.5 \mu \mathrm{m}$. From Figure 8, our two experimental methods predict a transition from mantle to core dominated deformation and the HREBSD slope agrees well with the grain core single crystal behavior, thereby assuming low-defect single crystal behavior in the core. [9] Dislocation densities peak within the mantle region as a consequence of excess GND's generated to satisfy the grain deformation gradient. However, varying the grain size would yield a change in the deformation gradient and hence the mantle dislocation density. For example, transitioning from $2 \mu \mathrm{m}$ to $20 \mu \mathrm{m}$ grain sizes decreases the theoretical mantle dislocation density by an order of magnitude, from $3 \times 10^{11} \mathrm{~cm}^{-2}$ to $3 \times 10^{10} \mathrm{~cm}^{-2}$. Therefore, the transition from mantle-dominated (fine grain) to core-dominant (coarse-grain) behavior is marked by the theoretical mantle mobile dislocation density limit approaching that of the core.

For the present case, creep accommodation by mantle deformation suggests the cores remain rigid-plastic and continuity is preserved by additional shear displacement accommodated by emitting dislocations from the grain boundary into parallel adjacent zones. [9] Additionally, normal displacements are accommodated through formation of dislocation pairs across the boundaries penetrating the mantle, into the core. [9] The former accommodation process follows the requirement for minimizing the mantle-core dislocation 
interactions proposed by Gifkens [7] and promotes grain rotations from shear displacements. [8] [38] Prior to Gifken's model, Ashby [9] , observed a grain size dependence, supporting the role of the mantle regions described by Gifkens [7] and Equation 5. Core-dominated deformation infrequently occurs compared with predominately low defect grains with mantle accommodation.

\section{Conclusion}

Mantle-core dislocation deformation was shown to accommodate $\mathrm{ZrB}_{2}$ grain boundary sliding for our Z20SB composite. High Resolution Electron Backscatter Diffraction (HREBSD) and Indentation Deformation Mapping (IDM) experiments were used for independently probing the deformation characteristics of the mantle and core regions in the context of a single crystal deformation (Orowan) model. Mantle geometrically necessary dislocation (GND) densities were one order of magnitude larger than those from the hot-pressed condition and in the crept grain core.

\section{Acknowledgements}

The authors gratefully acknowledge AFOSR program monitor Ali Sayir, under grant \# FA9550-09-1-0200, and DOE grant DE-SC0012587 for support of this work. Appreciation goes to Professors Bill Fahrenholtz and Greg Hilmas and Dr. Eric Neuman at the Missouri University of Science and Technology, Rolla, MO for providing the materials used in this study. Extended appreciation goes to Professor Greg Rohrer, at Carnegie Mellon University, for a critical review of this work and valuable recommendations.

\section{References}

[1] E L Courtright, H C Graham, A P Katz, and R J Kerans, "Ultrhigh Temperature Assessemnt Study-Ceramic Matrix Composites," Writgh-Patterson Air Force Base, Ohio, WL-TR-91-4061 1992.

[2] W.G. Fahrenholtz, G.E. Hilmas, I.G. Talmy, and J.A. Zaykoski, "Refractory Diborides of Zirconium and Hafnium," Journal of the American Ceramic Society, vol. 90, pp. 1347-1363, 
2007.

[3] L Kaufman and E V Clougherty, "Investigation of Boride Compounds for very High Temperature Applications," ManLabs Inc., Cambridge, RTD-TDR-63-4096 Part 11963.

[4] M W Bird, R P Aune, F Yu, P F Becher, and K W White, "Creep Behaviro of a Zirconium Diboride-Silicon Carbide Composite," Journal of the European Ceramic Society, 2013.

[5] T G Langdon, "Grain Boundary Sliding as a Deformation Mechanism during Creep," Philosophical Magazine, vol. 22, pp. 689-700, 1970.

[6] T G Langdon, "A Unified Approach to Grain Boundary Sliding in Creep and Superplasticity," Acta Metallurgica Materiala, vol. 42, no. 7, pp. 2437-2443, 1994.

[7] R C Gifkins, "Grain-Boundary Sliding and its Accommodation During Creep and Superplasticity," Metallurgical Transactions A, pp. 1225-1232, 1976.

[8] R C Gifkins, "Grain Rearrangements During Superplastic Deformation," Journal of Materials Science, pp. 1926-1936, 1978.

[9] M F Ashby, "The Deformation of Plastically Non-homogenous Materials," Philosophical magazine, vol. 21, no. 170, pp. 399-424, 1970.

[10] Marc de Graeff, Introduction to Conventional Transmission Electron Microscopy.: Cambridge University Press, 2003.

[11] B L Adams, "Orientation imaging microscopy: Emergin and future applications," Ultramicroscopy, vol. 67, pp. 11-17, 1997.

[12] C J Gardner, B L Adams, J Basinger, and D T Fullwood, "EBSD-based continuum dislocation microscopy," Interantion Journal of Plasticity , vol. 26, pp. 1234-1247, 2010.

[13] A J Wilkinson, G Meaden, and D J Dingley, "High-resolution elastic strain measurement form electron backscatter diffraction patterns: New levels of sensitivity," Ultramicroscopy, vol. 106, pp. 307-313, 2006.

[14] W Pantleon, "Resolving the geometrically necessary dislocation content by convental electron backscattering diffraction," Scripta Materialia, vol. 58, pp. 994-997, 2008.

[15] J F Nye, "Some Geometrical Relations in Dislocated Crystals," Acta Metallurgica, vol. 1, pp. 153-162, 1953.

[16] E Kroner, "Continuum theory of dislocations and self-stresses," Ergebnisse der Angewandten Mathematik, vol. 5, pp. 1327-1347, 1958.

[17] T J Ruggles and D T Fullwood, "Estimations of Bulk Geometrically Necessary Dislocation Density Using High Resolution EBSD," Ultramicroscopy, vol. 133, pp. 8-15, 2013.

[18] R E Reed-Hill and R Abbaschian, "Dislocations and Plastic Deformation," in Physical Metallurgy Principles, 3rd ed. Boston, MA: PWS Publishing Company , 1994, pp. 124-167.

[19] T G Langdon, "Grain-boundary sliding during creep of MgO," in Pacific Coast Regional Meeting, The American Ceramic Society, San Francisco, 1974, pp. 92-93. 
[20] A L Chamberlain, W G Fahrenholtz, G E Hilmas, and D T Ellerby, "High-Strength Zirconium Diboride-Based Ceramics," Journal of the American Ceramic Society, pp. 1170-1172, 2004.

[21] M W Bird, R P Aune, A F Thomas, P F Becher, and K W White, "Temperature-dependent mechanical and long crack behavior of zirconium diboride-silicon carbide composite," Journal of the European Ceramic Society, vol. In Press, pp. 3453-3462, 2012.

[22] F J Humphreys, "Characteristion of fine-scale microstrcutures by electron backscatter diffaction (EBSD)," Scripta Materiallia, vol. 51, pp. 771-776, 2004.

[23] EDAX, "TSL Orientation Imagaing Microscopy Anslysis 6 Manual," Materials Analysis Division, AMETEK, Inc., Software Manual 2011.

[24] A J Schwartz and M Kumar, Electron backscatter diffraction in materials science, 2 nd ed. New York, NY: Springer, 2009.

[25] K Z Troost, P V D Sluis, and D J Gravesteijn, "Microscale elastic-strain determination by backscatter Kikuchi diffraction in the scanning electron microscope," Applied Physics Letters, vol. 62, pp. 1110-1112, 1993.

[26] C Landon, B Adams, and J Kacher, "High resolution methods for characterizing mesoscale dislocation structures," Journal of Engineering Materials and Technology, vol. 130, pp. 4045, 2008.

[27] J Kacher, C Landon, B L Adams, and D T Fullwood, "Bragg's Law Diffraction Simulations for Electron Backscatter Diffraction Analysis," Ultramicroscopy, vol. 109, pp. 1148-1156, 2009.

[28] C J Gardner, B L Adams, and D T Fullwood, "EBSD-based continuum dislocation microscopy," International Journal of Plasticity, vol. 26, pp. 1234-1247, 2010.

[29] F Goutier, G Trolliard, S Valette, A Maitre, and C Estournes, "Role of impruities on spark plasma sintering of ZrCx-ZrB2 composites," Journal of the European Ceramic Society, vol. 28, pp. 671-678, 2008.

[30] S K Mishra, S Das, and L C Pathak, "Defect structure in zirconium diboride powder prepared by self-propagating high-temperature synthesis," Materials Science and Engineering, vol. A364, pp. 249-255, 2004.

[31] T Mizuguchi, S-Q Guo, and Y Kagawa, "Transmission Electron Microscopy Characterization of Hot-Pressed ZrB2 with MoSi2 Additive," Journal of the American Ceramic Society, vol. 92, no. 5, pp. 1145-48, 2009.

[32] T Mizuguchi, S Guo, and Y Kagawa, "Transmission electron microscopy characterization of spark plasma sintered ZrB2 ceramic," Ceramics International, vol. 36, pp. 943-46, 2010.

[33] J S Haggerty and D W Lee, "Plastic Deformation of ZrB2 Single Cyrstals," J. Amer. Ceram. Soc., vol. 54, no. 11, pp. 572-576, 1971.

[34] A Ball and M M Hutchinson, "Superplasticity in the Aluminum-Zinc Eutectoid," Metal Science Journal, pp. 1959-1969, 1968.

[35] M F Ashby and R A Verrall, "Diffusion-Accommodated Flow and Superplasticity," Acta 
Metallurgica, vol. 21, pp. 149-63, February 1973.

[36] M W Bird, P F Becher, and K W White, "Grain Rotation and Trnaslation Contribute Substantially to Flexure Creep of a Zirconium Diboride Silicon Carbide Composite," In-Press, 2013.

[37] R C Gifkins and T G Langdon, "Comments on Theories of Structural Superplasticity," Materials Science and Engineering, pp. 27-33, 1978.

[38] S B Biner, "The role of grain boundary sliding on creep deformation characteristics of discontinuous reinforced composites," in Plastic Deformation of Ceramics, R.C. Bradt et al., Ed. New York: Plenum Press, 1995, pp. 495-505. 


\section{Figures}

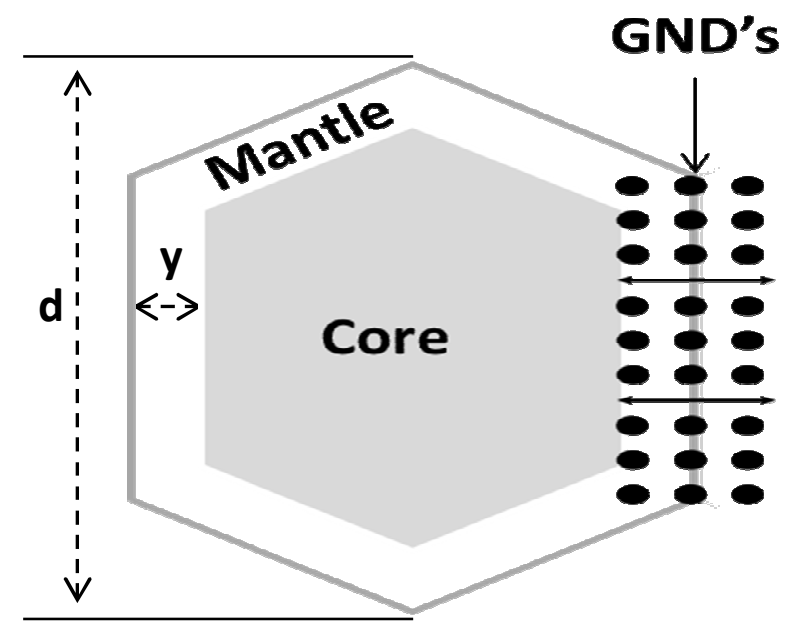

Figure 1:Illustration of mantle-core grain deformation and the position of geometrically necessary dislocations (GND's), required for continuity, from the inherent deformation gradient. Recreated from Ref [ 7]. Dimensions $d$ and y are the grain size and mantle thickness, respectively. 

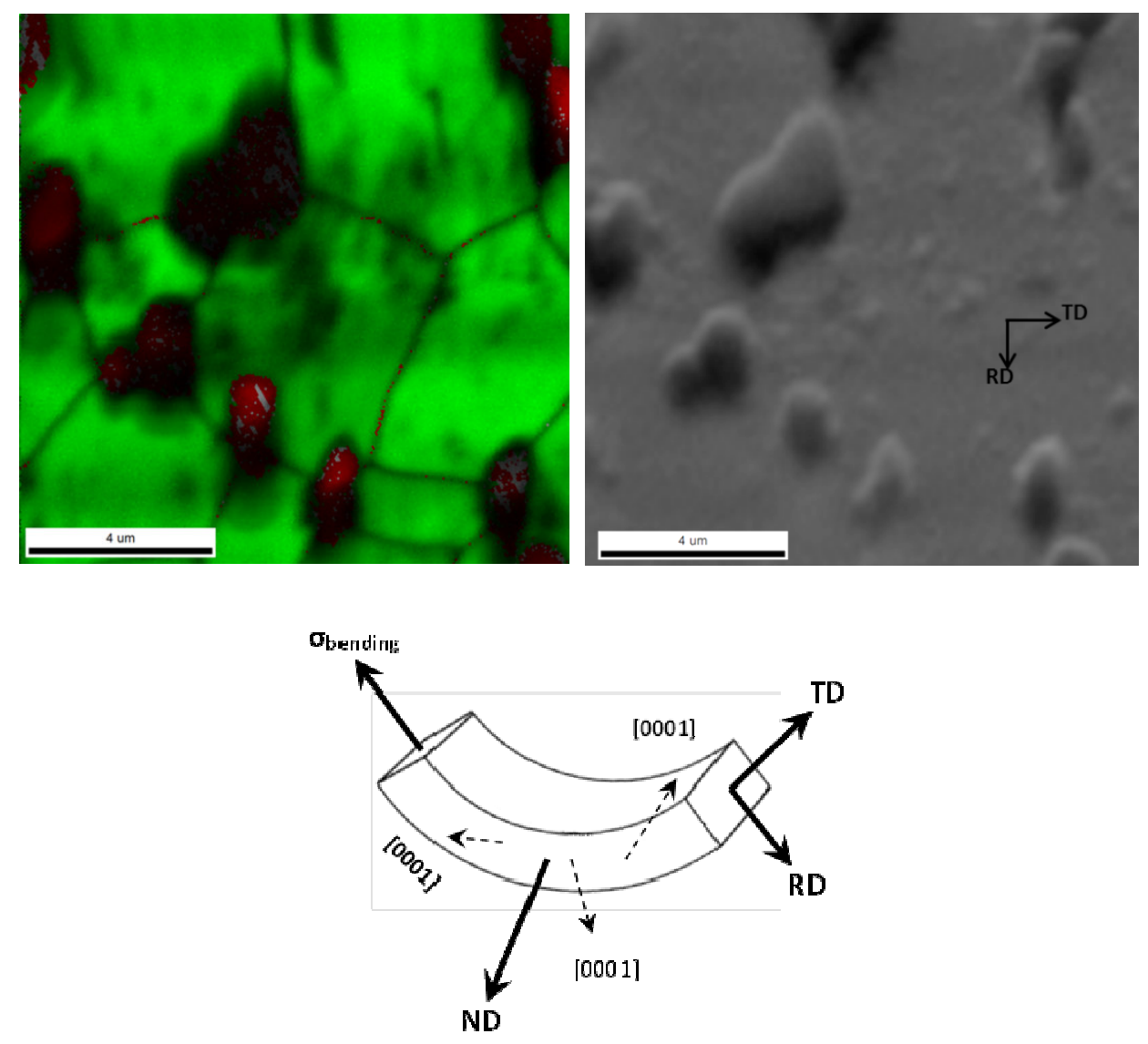

Figure 2: EBSD reconstruction map highlighting the SiC phase (red) and ZrB2 phase (green) based on confidence index (left) and the actual SEM image (right). RD, TD and ND are sample space default directions with TD parallel to the bending stress axis (bottom). 

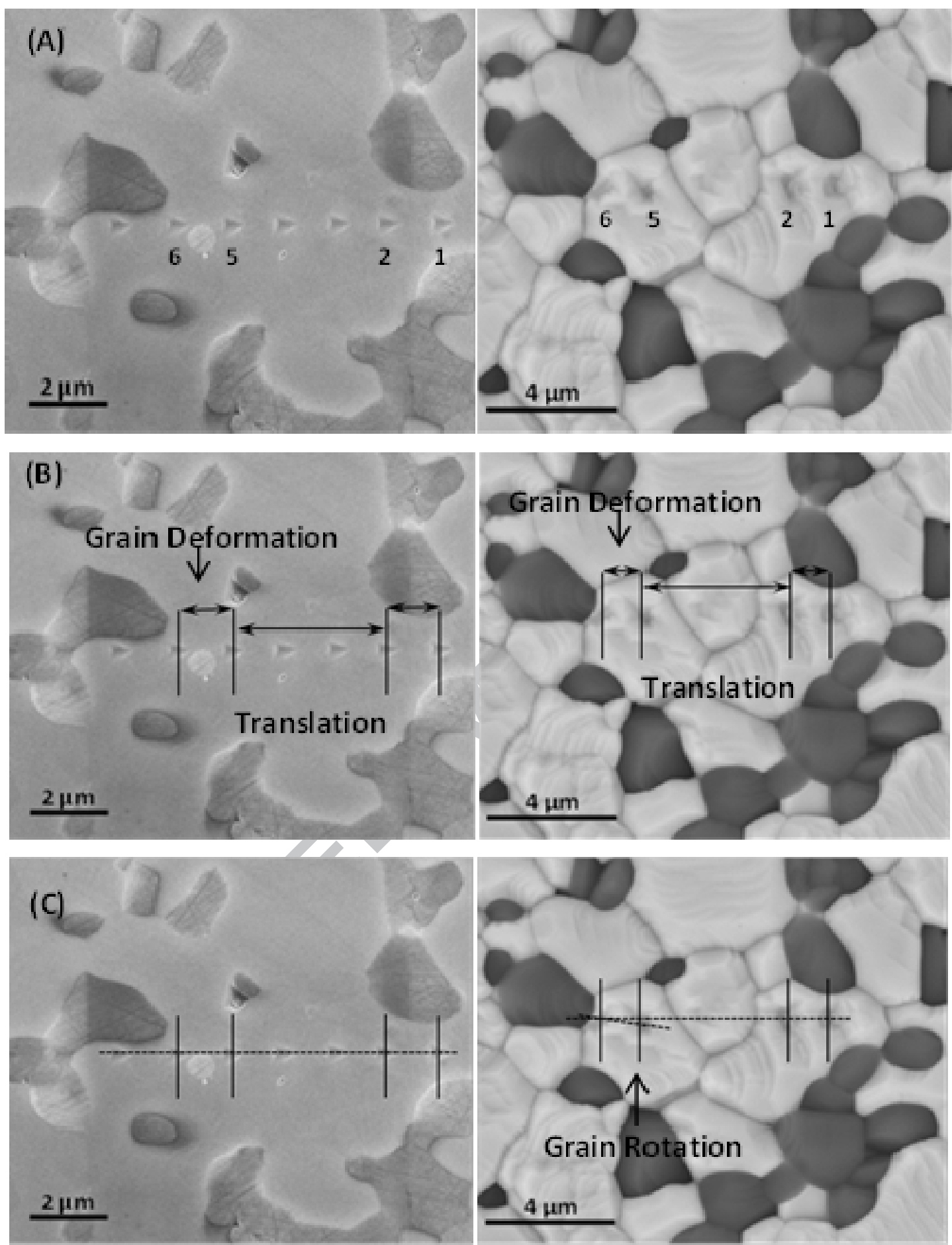

Figure 3: Secondary SEM image of the un-crept state highlighting cube corner nano-indents (left) and high resolution backscatter images of the as-crept surface (right). Indents were labeled according to position for reference between initial and final conditions (a). Grain deformation and translation displacements are illustrated in (b) and grain rotation shown in (c). Vertical dotted and solid lines are indent shape boundaries based on threshold imaging and the average indent shape position, respectively 


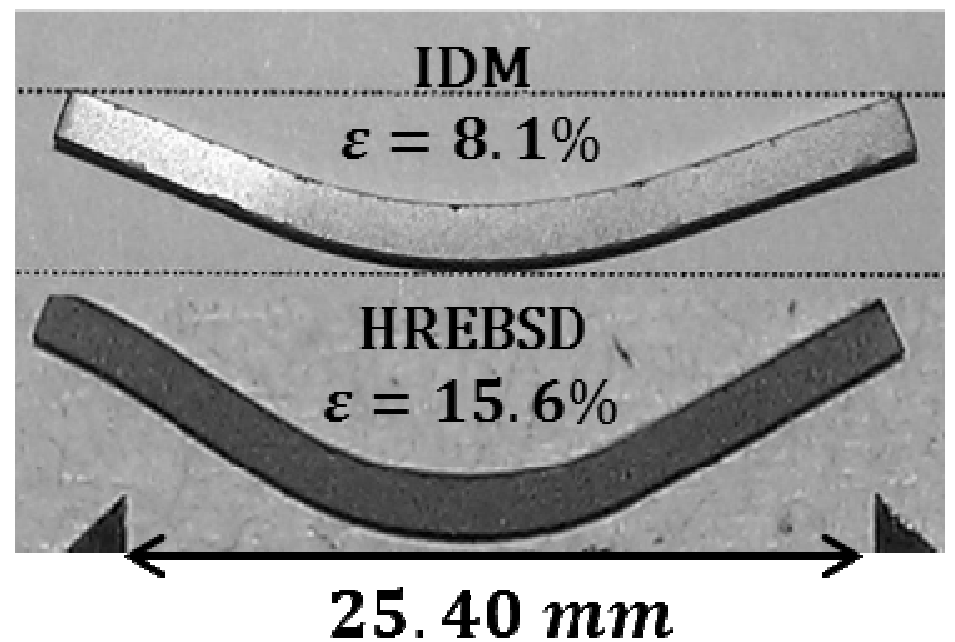

Figure 4: High strain Z20SB creep specimen examples used for IDM and HREBSD measurements. Specimens were crept at $1800 \mathrm{C}, 16 \mathrm{MPa}$. The IDM and HREBSD creep experiments wer conducted for 5 and $7.75 \mathrm{hrs}$, respectively. The Outer-fiber tensile strains of $8.1 \%$ and $15.6 \%$ for the IDM and HREBSD specimens, respectively, were determined using optical methods. 

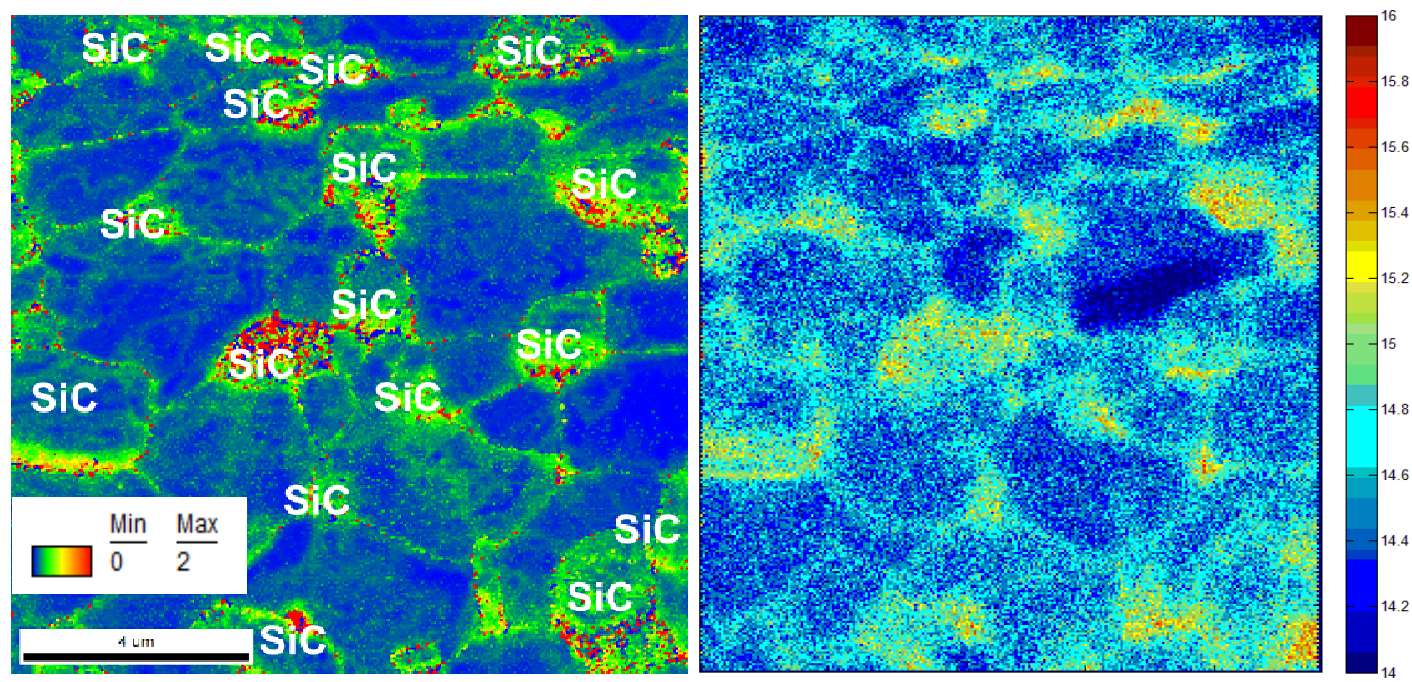

Figure 5: Compression bending region KAM (left) and HREBSD GND concentration rendering (right) regions. SiC particles are labeled for reference and the GND color map is in $\log _{10} \mathrm{~m}^{-2}$ units. 

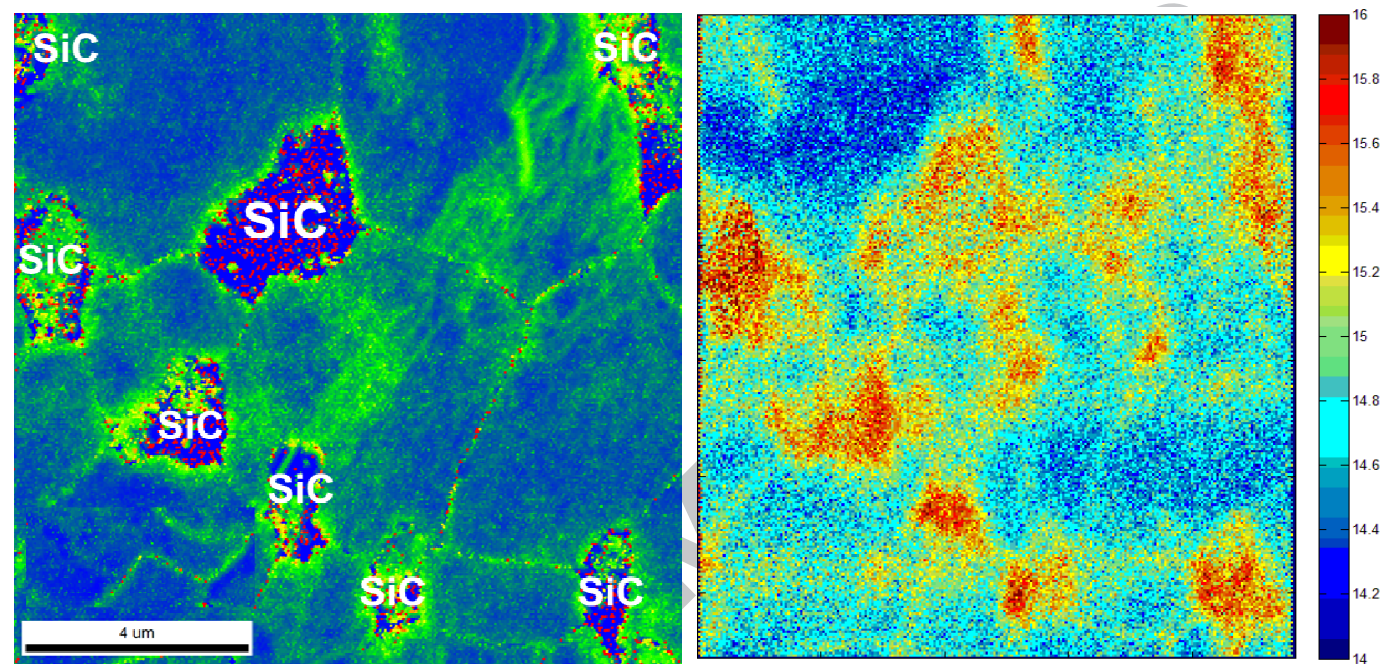

Figure 6: Tension bending region KAM (left) and HREBSD GND concentration rendering (right) regions. SiC particles are labeled for reference and the GND color map is in $\log _{10} \mathrm{~m}^{-2}$ units. 

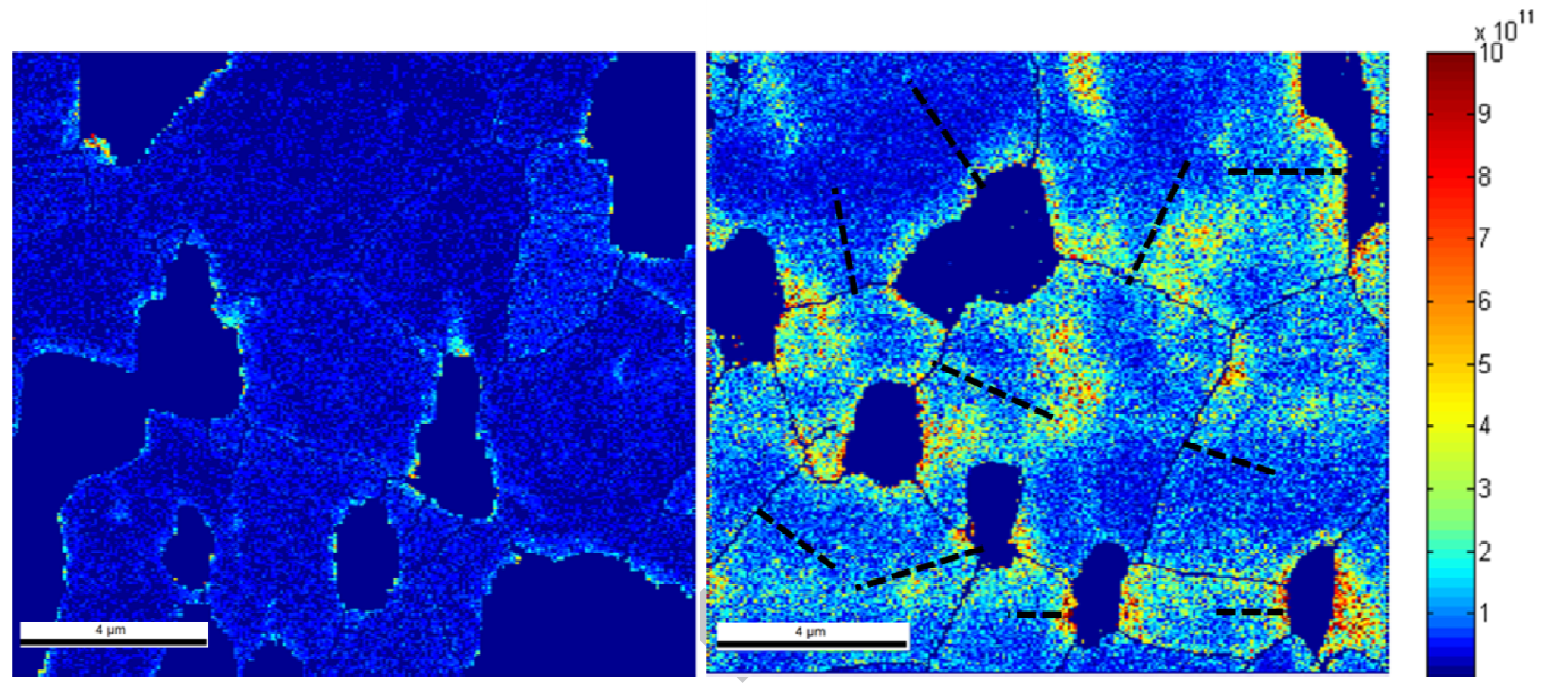

Figure 7: Revised HREBSD Hot pressed (left) and tensile zone (right) featuring ZrB ${ }_{2}$ GND density spatial distributions in $\mathrm{cm}^{-2}$. SiC particles are the solid phases. Hashed lines illustrate the GND random profile trace positions used for constructing the HREBSD Orowan model. 


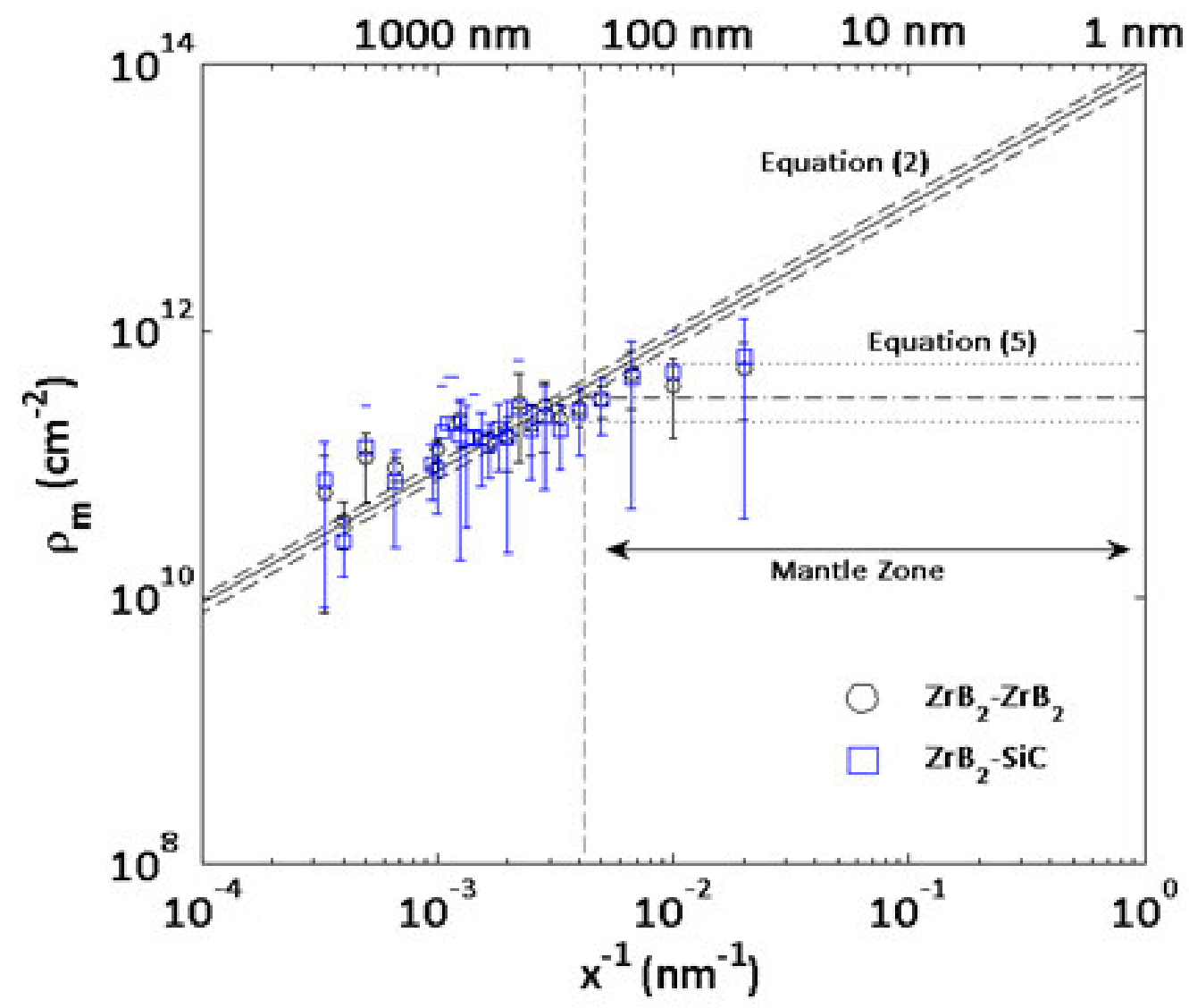

Figure 8: Equation 2, $\gamma=\rho_{m} b \bar{x}$, (solid line) with the mean 95\% confidence bounds (dashed lines) overlaid with Equation 5 (double hash line) and range (dotted lines) GND densities from the HREBSD grain size distribution. HREBSD GND densities for $\mathrm{ZrB}_{2}-\mathrm{SiC}$ (square) and $\mathrm{ZrB}_{2}-\mathrm{ZrB}_{2}$ (circle) boundaries are included for reference. Error bars represent the standard deviations of GND densities for all line profiles. 


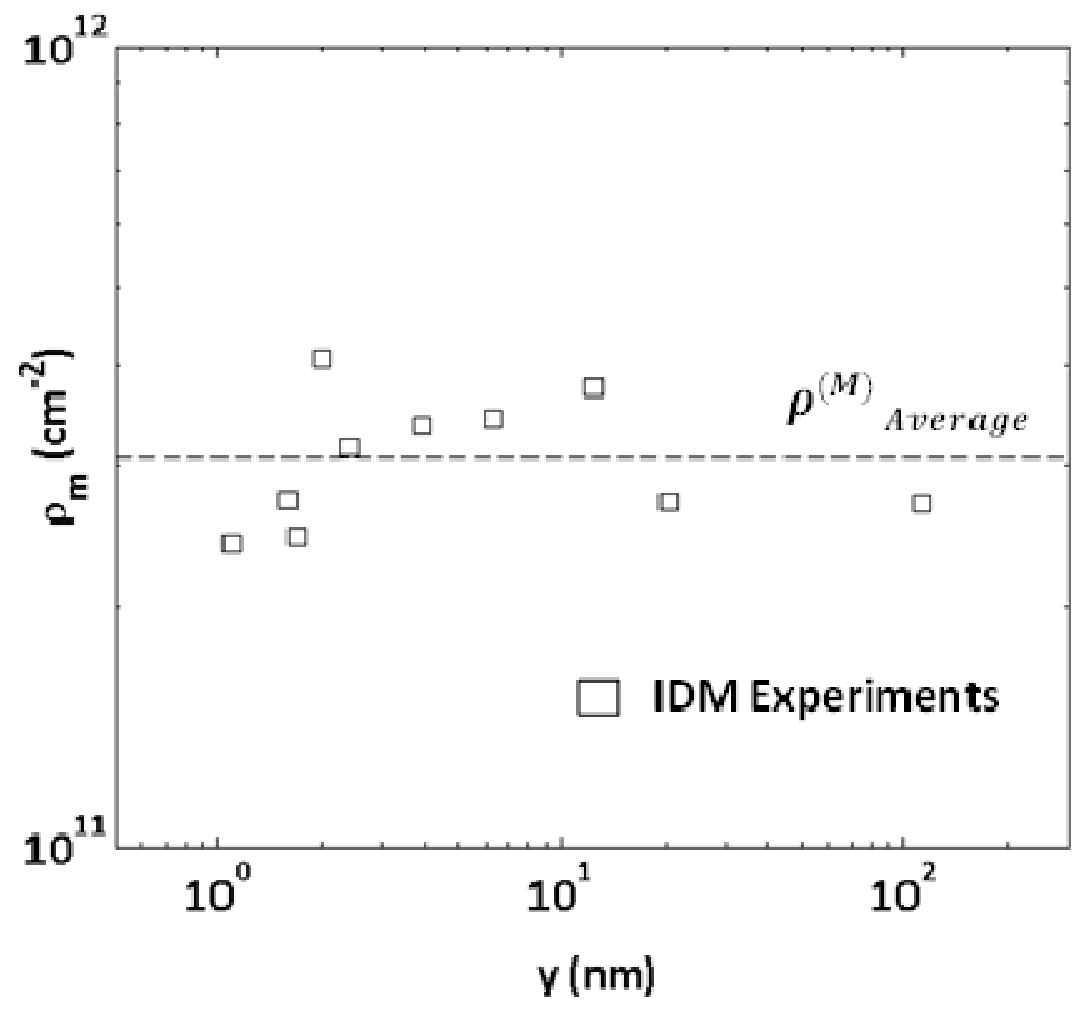

Figure 9: $\rho_{m}$ (Equation 5, $\bar{x}=y$ ) and y (Equation 3) calculated using the grain rotation results, of individual grains, from the IDM experiments. The hashed line represents the grand average $\rho_{m}$ of all individual grains shown. 


\section{Tables}

Table 1: Select IDM grain size $(d)$, grain core deformation strains $\left(\varepsilon_{\text {Core }} \pm 0.01 \mu m / \mu m\right)$ and grain rotation angles $\left(\theta \pm 0.14^{\circ}\right)$

\begin{tabular}{ccc}
\hline \hline$d(\mu m)$ & $\varepsilon_{\text {Core }}$ & $\theta$ \\
\hline \hline 4.08 & 0.007 & 0 \\
4.08 & -0.109 & 0 \\
4.08 & 0.013 & 0.11 \\
4.08 & -0.118 & 0.38 \\
4.84 & -0.050 & 0.51 \\
4.24 & 0.007 & 0.57 \\
5.34 & 0.000 & 1.00 \\
3.41 & -0.040 & 1.03 \\
4.06 & 0.000 & 1.48 \\
5.49 & -0.112 & 1.53 \\
5.35 & -0.066 & 1.99 \\
4.84 & 0.087 & 2.17 \\
4.12 & -0.014 & 2.73 \\
4.56 & 0.000 & 2.86 \\
3.21 & 0.072 & 2.98 \\
3.93 & -0.120 & 3.42 \\
3.93 & 0.000 & 3.42 \\
3.81 & 0.000 & 4.30 \\
3.35 & 0.000 & 4.56 \\
3.82 & 0.079 & 4.86 \\
4.09 & 0.000 & 5.44 \\
3.76 & 0.000 & 6.07 \\
3.47 & 0.029 & 6.96 \\
4.85 & -0.022 & 7.38 \\
4.85 & 0.000 & 7.38 \\
4.87 & -0.056 & 16.93 \\
2.3 & 0.000 & 37.41 \\
& & \\
\hline
\end{tabular}

